\title{
Achieving High Data Compression of Self-Similar Satellite Images using Fractal
}

\author{
Wee Meng Woon, Anthony Tung Shuen Ho, Tao Yu, Siu Chung Tam, \\ Siong Chai Tan and Lian Teck Yap \\ Nanyang Technological University, \\ School of Electrical and Electronic Engineering, Nanyang Avenue, Singapore 639798 \\ Tel: (65)-793-7726 / Fax: (65)-793-7790 / E-mail: p7330048g@ntu.edu.sg
}

\begin{abstract}
In this paper, we examine and describe an implementation of a fractal compression method on optical satellite images. The basic principle is that an image can be reconstructed by using the self similarities in the image itself. The satellite image is first partitioned into a set of non-overlapping ranges. For each range, a "best matching" domain block would be found and a set of affine transformation would be performed. The compression would be obtained by storing only the descriptions of this transformation.

The fractal compression method is implemented on two images, a complicated city image and a simple coastal area image. Using a fractal algorithm, the data integrity of the coastal area image was maintained with a Peak Signal-to-Noise Ratio (PSNR) of approximately 34.9dB while achieving a compression ratio of 172:1. A novel approach of using a combination of fractal and wavelet algorithms for data compression will also be described.
\end{abstract}

\section{INTRODUCTION}

Digital images associated with satellite remote sensing posses the characteristics of being data intensive. Huge amount of data would require much storage space and longer transmission time of data. In order to solve these storage space and time constraints, data compression has always been actively researched upon by the remote sensing community.

The term fractal was first used by Benoit Mandelbrot to designate objects that are self-similar at different scales. Mandelbrot had observed that complex images could be obtained from simple formulas. Given a formula, it is relatively easy to derive the corresponding image. Bansley, another researcher, implemented this concept to image compression. Instead of representing the image as a long sequence of pixel value, the image can be reconstructed from a formula, which can be encoded in a much smaller number of bytes. This form of compression is termed as fractal compression. [1]

The focus in this paper is the examination and implementation of a simple fractal algorithm on satellite images. In this method, the redundancy of the digital image is assumed to be exploitable through self-transformability on a block-wise basis, i.e. the image can be built of transformed copies of parts of itself. The key behind fractal image compression is self-similarity in images. [2]

This fractal algorithm is implemented on two images, one being a complicated image and the other being a simple image with many similar segments. This implementation demonstrates the effectiveness of the fractal compression method on satellite images with many similar segments. Although direct implementation of the fractal compression algorithm does not provide a universal solution to all satellite images, it could prove useful if combined with wavelet transformations. [3]

\section{SATELLITE IMAGE COMPRESSION USING FRACTAL ALGORITHM}

Fractal image compression is a compression scheme that encodes one part of an image by other parts of the image through some transformation techniques. The image in this fractal compression method is firstly partitioned into a number of blocks of size $2 \mathrm{~N} \times 2 \mathrm{~N}$ pixels called domain blocks and a number of non-overlapping blocks of size $\mathrm{N} \times \mathrm{N}$ pixels called range blocks. For each non-overlapping range, the algorithm aims to find the domain block and transformation that is the best match of the range.

One major setback of fractal compression is the complicated computations required, resulting to very slow compression operations. To speed up the compression process, some cumulative tables are created for the range and domain data. Another significant speed improvement is achieved by avoiding domain-range comparisons that have little probability of providing a good match. In the algorithm, the range and domain blocks are categorized to different classes and comparisons are done to members of the same class. The class is determined by the ordering of the image brightness in the four quadrants of the range or domain.

After the initial computation of the image is completed, a good match for a non-overlapping range block and a domain block is attempted. If there is no domain block could match the range block based on a specified quality value, the range is further split into 4 ranges. This process is repeated recursively until the minimum size of the range block or the specified quality value is attained.

To access the similarity between a domain block and a range block, the best possible mapping from a domain block and range block is to be found. Affine transformations are used for this purpose.

An affine map is defined as follows,

$$
W i\left(\begin{array}{l}
x \\
y \\
z
\end{array}\right)=\left(\begin{array}{ccc}
a_{i, 1} & a_{i, 2} & 0 \\
a_{i, 3} & a_{i, 4} & 0 \\
0 & 0 & c_{i}
\end{array}\right)\left(\begin{array}{l}
x \\
y \\
z
\end{array}\right)+\left(\begin{array}{l}
d_{i, 1} \\
d_{i, 2} \\
b_{i}
\end{array}\right)
$$

where $W i$ is the mapping of a domain block to a range block, $x$ and $y$ are the spatial components of a domain block, $z$ 
is the luminance component of a domain block, $a$ is the scaling factor, $d$ is the translation factor, $c$ is the contrast factor, and $b$ is the brightness factor.

The specified quality value or the best affine map from a range block and a domain block is found by computing the sum of squared errors between a range block and a mapped domain block.

$$
E(c, b)=\sum_{i=1}^{n}\left(c d_{i}+b-r_{i}\right)^{2}
$$

where $E$ is the sum of squared errors, $d i$ is the pixel value in a domain, $r i$ is the pixel value, $\mathrm{n}$ is the number of pixels in a range block, $c$ (contrast) could be obtained from,

$$
C=\frac{n \sum_{i=1}^{n} r_{i} d_{i}-\sum_{i=1}^{n} d_{i} \sum_{i=1}^{n} r_{i}}{n \sum_{i=1}^{n} d_{i}^{2}-\sum_{i=1}^{n} d_{i} \sum_{i=1}^{n} d_{i}}
$$

and $b$ (brightness) could be obtained from,

$$
b=\frac{\sum_{i=1}^{n} r_{i}-c \sum_{i=1}^{n} d_{i}}{n}
$$

In the decompression module, the encoded affine maps are first read in. The decoder then starts with a random initial image and undertakes a few iterations. For each iteration, all maps are applied to the current image.

Therefore, in the fractal compression method, the encoding of the total number of affine transformations requires far fewer bits than enumerating all the pixel values, resulting to data compression. [2]

\section{EXPERIMENTAL RESULTS}

In this section, we present some experimental results of satellite image compression by applying the fractal algorithm on two satellite images, a city area and a coastal area. By varying the quality value, different compression ratio with corresponding fidelity value could be obtained.

In a digital image processing system, it is usually necessary to provide some form of performance comparison between an original image and a processed image. One approach is to specify image fidelity in terms of the discrete samples of the images. One common discrete image fidelity measures expressed in decibel form is the Peak Signal To Noise Ratio (PSNR). Besides knowing the image fidelity measure, the most important information involving compression is the information on how many times the image is being compressed. The compression measure I used for comparison is the Compression Ratio (CR).

The Peak Signal to Noise Ratio (PSNR) is defined as follows,

PSNR $=-10 \log \left[\Sigma(\mathrm{V}-\mathrm{V} \text { original })^{2} / \Sigma 255^{2}\right]$

where Voriginal is a pixel value of the original image, $\mathrm{V}$ is a pixel value of the reconstructed image.

Figure 1 presents some experimental results in a graphical manner, comparing PSNR with CR for the two different satellite images.

Figure 2 shows reconstructed images with different compression ratios and fidelity values. A compression ratio of $11: 1$ with a PSNR of $25.03 \mathrm{db}$ could be obtained from a satellite image of a city while a compression ratio of 173:1 with a PSNR of $34.9 \mathrm{~dB}$ could be obtained from a satellite image of a coastal area.

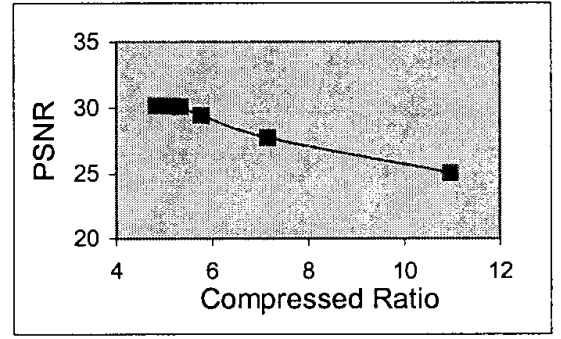

a) Satellite image of a City Area

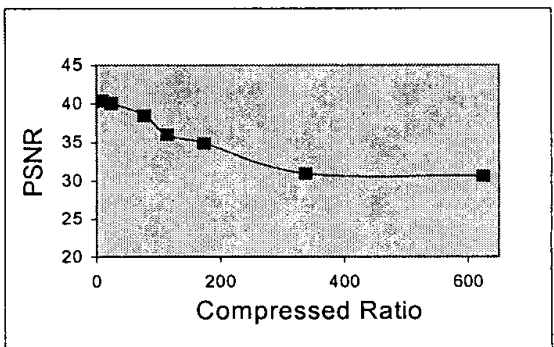

b) Satellite image of a Coastal Area

Figure 1. Graphs of PSNR vs CR 


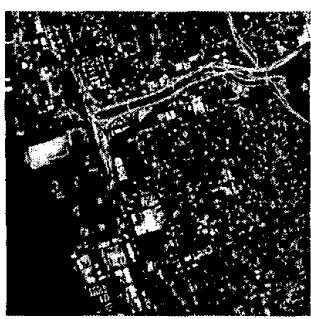

a) Original Satellite Image Of a City

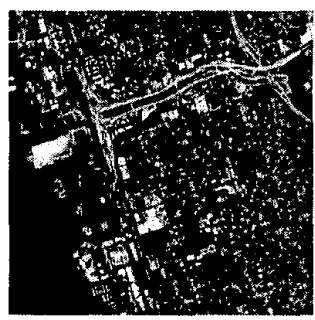

b) $\quad \mathrm{CR}=5: 1$

PSNR $=30.16 \mathrm{~dB}$

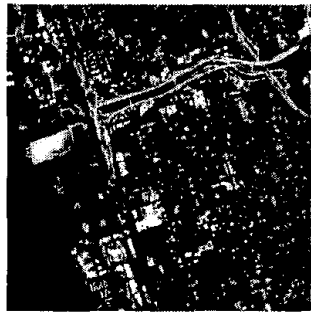

c) $\quad \mathrm{CR}=11: 1$ PSNR $=25.03 \mathrm{~dB}$

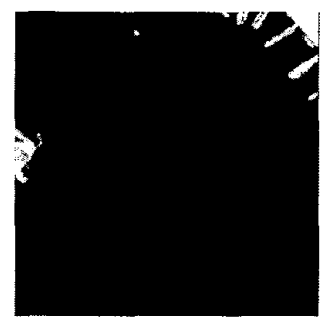

d) Original Satellite Image Of a Coastal Area

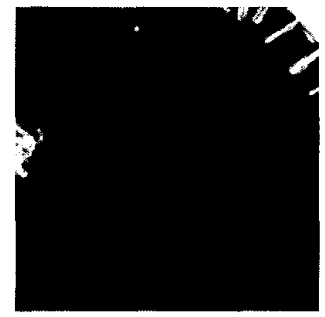

e) $\quad C R=9: 1$

PSNR $=40.39 \mathrm{~dB}$

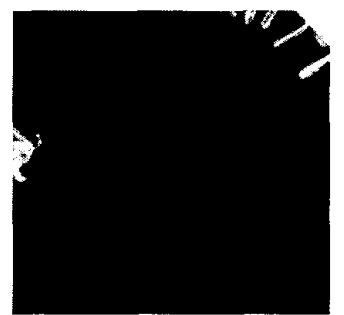

f) $\quad \mathrm{CR}=173: 1$ $\mathrm{PSNR}=34.9 \mathrm{~dB}$
Figure 2. Remote Sensing Image Compression Using Fractal algorithm

\section{DISCUSSIONS}

The experimental results show that the fractal compression method could achieve better results for images with more similarities such as the coastal area image.

The fractal compression algorithm could be improved by implementing it with bi-orthorgonal wavelet filters. Applying wavelet filters to an image will transform the image from a time domain into a frequency domain. In the initial iteration of the wavelet filter algorithm, the image is decomposed into one coarse and three detail components. After $\mathrm{N}$ decomposition iterations, there would be $3 \mathrm{~N}$ detail components and one coarse component of the original image. The coarse component of the image could be understood as a low-pass filtered sub-image and the detail components understood as high-pass filtered sub-images of the original image. Figure 3 shows an image which undergone 3 iterations with one coarse component and 9 detail components. It can be observed that the various detail components have similarities.

The coarse component of the image is first coded using adaptive differential pulse modulation method (ADPCM) while the fractal algorithm is implemented on the detail components. Higher compression ratio with lower PSNR results would be attained with an image undergoing more wavelet filters iterations. [3]

\section{CONCLUSION}

In this paper, a fractal compression algorithm was described and successfully implemented on two satellite images, a complicated and a simple image. Using the fractal compression algorithm, it was found that the compression ratio could reach up to $173: 1$ while maintaining a PSNR of $34.9 \mathrm{~dB}$ for a coastal area image. As for the city area image, compression ratio could reach up to $11: 1$ while maintaining a PSNR of $25.03 \mathrm{~dB}$. An improvement to the fractal compression method by combining fractals and wavelets has also been described.

\section{REFERENCES}

[1] Michael F.Barnsley, "Fractals Everywhere", Academic Press INC, 1988 .

[2] Mark Nelson, "The Data Compression Book", M\&T Books, 1996.

[3] T Yu, A.T.S Ho, “ A Novel Hybrid Bi-orthogonal Wavelet/ADPCM Algorithm for very Low Bit Rate Satellite Image Compression ", IEEE/IGARSS, 1999.

[4] Heinz-Otto Peitgen, Dietmar saupe, "Chaos \& Fractals : New Frontiers of Science, Springer Verlag, 1992.

[5] Yuval Fisher, "Fractal Image Compression", SpringerVerlag, 1995.

[6] A.T.S Ho, T Yu, "Improving Vector Quantization of Satellite Images Through the Application of Bi-orthogonal Wavelets", IEEE/IGARSS, 1999.

[7] Burrus C.S, "Introduction to wavelets and wavelet transform: a primer", Prentice Hall, 1998.

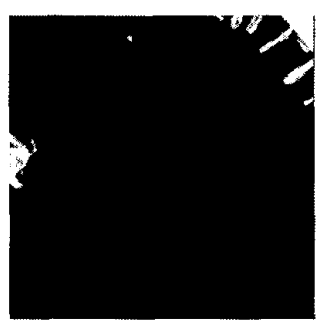

a) Original

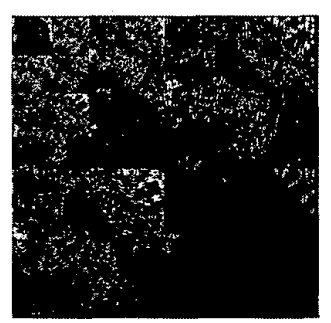

b)Wavelet filters applied
Figure 3. Application of wavelet filter on a Satellite Image of a coastal area 